\section{P130 PROGRESS FOR PROVIDERS: END OF LIFE - CHECKING YOUR ORGANISATION'S PROGRESS IN DELIVERING PERSONALISED SUPPORT}

${ }^{1}$ Philip Ball, ${ }^{2}$ Helen Sanderson, ${ }^{3}$ Tracy Meyerhoff, ${ }^{4}$ Nichola Monks, ${ }^{4}$ Anne Torkington. ${ }^{1}$ Sue Ryder, Peterborough, UK, ${ }^{2}$ Helen Sanderson Associates, Stockport, UK, ${ }^{3}$ Hull City Council, UK, ${ }^{4}$ St Ann's Hospice, Greater Manchester, UK

10.1136/bmjspcare-2013-000591.152

Introduction The hospice movement often uses the term 'holistic care' to describe what it provides, with it being applied differently depending on the care setting. Personalisation could be considered another version of holistic care. Based on this we took the opportunity of bringing both holistic care and personalisation together, to explore what this means in practice for end of life care.

Progress for Providers is a recognised series of self-assessments, to enable providers to see how they are doing in delivering personalisation. There's not something of this nature already available to hospice managers. Personalisation has primarily been a term used by social care, with government plans to integrate health and social care by 2018 , its key we start to embed personalisation to hospice care now.

Aims The aim is to enable hospice managers, and anyone involved in supporting people at end of life, to know what personalisation looks like in practice and to introduce person-centred thinking tools that can add to the way that hospices can deliver truly personalised services.

Approach used The authors met to produce the self-assessment tool using their knowledge, key reports and other sources of information to inform the statements of each area of assessment. The tool will be sent out for comment, refined and tested during the summer of 2013, to be launched in October.

Outcomes and applications to hospice practice The use of Progress for Providers: End of Life offers an opportunity to critically examine local practice with a view to taking personalisation further.

While the future of hospices has been considered by the Commission, making progress in personalisation is another way to look at service provision to ensure it meets the needs of the people we support.

\section{P131 GARDEN HOUSE HOSPICE ADMISSION RESPONSE CRITERIA}

Augustina Ebiredelu. Garden House Hospice, Letchworth, UK

10.1136/bmjspcare-2013-000591.153

Introduction Referrals for admissions are received from health care professionals in the community and acute trust with GP's knowledge. Requests are assessed by the admission co-ordinator and a member of the Hospice Medical team on an individual basis, without discrimination and in accordance with the admission policy.

A tool has been adapted from Luton Community Service Palliative care response criteria. It is based on RED/AMBER/ GREEN (RAG) category model.

Aim The aim is to ensure a responsive service based on patient needs. It provides clinicians with a user-friendly tool to support decision making when prioritising request for admission to the Hospice.

Method An audit on the use of the tool was undertaken.
RAG tool covers 3 categories; End of life care, symptom management and Psychological/social support.

RED - Death expected within hours/days, Symptom control and Carer breakdown. Admission/bed offered within 24 hours

AMBER - Death expected within days/weeks, symptoms assessed but remain unresolved, Psychological support requiring regular observation. Patient is admitted within 3 days.

GREEN - Death expected within weeks/months. Respite for family. Admission offered if no red or amber patients are waiting.

The tool is used in conjunction with a list of questions when dealing with request for admission.

Using the criteria, admission was prioritised according to RAG tool and response time documented.

Results During a 12 months period, 202 patients were admitted to the IPU from home or from hospital. 140 of these admissions were considered to be RED requests, 47 were AMBER and 15 were GREEN.

- Admission between 2-24hrs $=140$

- Admission between $24 \mathrm{hrs}-36 \mathrm{hrs}=47$

- Admission between $36 \mathrm{hrs}$ and over $=15$

Summary The RAG tool was found to be successful in prioritising request for admission. Streamlining the process of admission meant that patients requiring urgent admission were prioritised therefore preventing possible hospital admission and achieving their Preferred Place of Care and Death.

\section{P132 TO ADMIT OR NOT TO ADMIT? - THE USE OF A WAITING LIST SCORING SYSTEM TO PRIORITISE ADMISSION REQUESTS}

Aruna Hodgson. Wigan \& Leigh Hospice, Wigan, UK

\subsection{6/bmjspcare-2013-000591.154}

The problem A hospice inpatient unit was receiving more requests for admission than it could meet. Patients who were felt to need urgent admission would tend to be offered a bed ahead of those whose need was thought to be less urgent. This could result in those with complex moderate intensity symptoms having to wait a considerable time for admission, leading to ongoing suffering and often an escalation in their needs.

The attempted solution A simple scoring system was introduced to assist with prioritisation. Referrers are asked to specify how urgently admission is required (Immediate $=$ within 24 hours, Soon $=$ within 3 days, Routine $=$ within 5 days). Patients are allocated a score for priority of need and then accrue points according to each working day they are on the waiting list. When a bed becomes available, it is allocated to the patient with most points. When several patients have the same number of points and only one bed is available, the team discusses the relative needs of each patient to decide who will be offered the bed. The outcome Despite training prior to the introduction of the scoring system, there was initially some inconsistency in the way referrers assessed urgency, with a number of patients being listed as "Immediate" inappropriately. Following further discussion and education, this has improved. The system now works well and although demand for beds often still exceeds availability, the system allows them to be allocated in a fair and equitable way and has reduced the time taken to decide which patient (s) will be admitted when there are several on the waiting list. The system has attracted interest from other local hospices who are struggling with the same problem of over demand for beds. 